\section{Rotatividade dos trabalhadores de enfermagem nos hospitais do Município de Ribeirão Preto}

\author{
Maria Luiza Anselmi, ${ }^{1}$ \\ Emília Luigia Saporiti Angerami ${ }^{1}$ \\ e Elizabeth Laus Ribas Gomes ${ }^{1}$
}

\section{RESUMO}

Este estudo, desenvolvido em 1990, teve como objetivo a mensuração e a análise da rotatividade dos trabalhadores de enfermagem alocados nos nove hospitais gerais do Municipio de Ribeirão Preto, Estado de São Paulo, Brasil. A população investigada constituiu-se de enfermeiros, auxiliares de enfermagem, técnicos de enfermagem e atendentes de enfermagem admitidos ou demitidos entre $1^{\circ}$ de janeiro e 31 de dezembro de 1990. O acompanhamento foi feito através de consulta mensal junto à seção de pessoal dos hospitais. A rotatividade foi quantificada com a aplicação de dois indicadores de rotatividade, a taxa líquida de substituição e a mediana de tempo de serviço do grupo de trabalhadores demitentes. Os resultados evidenciaram o crescimento da força de trabalho em enfermagem em Ribeirão Preto no ano de 1990. Porém, também ficou evidente a elevada rotatividade, principalmente nos hospitais filantrópicos e privados investigados, com taxas líquidas de substituição de $32 \%$ e $39 \%$ ao ano e mediana de tempo de serviço de 8 meses e 12 meses, respectivamente, o que poderia causar aumento de custos e comprometer a produtividade. O único hospital público investigado apresentou níveis mais baixos de rotatividade em comparação aos demais, com uma taxa líquida de substituição de $6 \%$ ao ano e mediana de tempo de serviço de 42 meses. As categorias de enfermeiro e atendente de enfermagem foram as mais substituídas e, conseqüentemente, as menos estáveis no emprego.

Escola de Enfermagem de Ribeirão Preto, Universidade de São Paulo, Avenida Bandeirantes, 3900, CEP 14040-902, Ribeirão Preto, SP, Brasil. Correspondência e pedidos de separatas devem ser enviados para Emília Luigia Saporiti Angerami neste endereço.
Nas décadas 70 e 80 e, principalmente, a partir de 1975, o mercado de trabalho em saúde se transformou em um dos grandes segmentos na divisão social do trabalho. Este segmento se expandiu significativamente dentro do processo de terceirização da economia e, neste sentido, vem se constituindo num pólo expressivo de absorção de trabalhadores e de geração de empregos $(1,2)$. A rotatividade dos trabalhadores é um movimento importante na dinâmica do mercado de trabalho em saúde. Porém, embora amplamente analisado em outros setores econômicos, no setor de saúde este é um movimento pouco estudado.

A rotatividade compreende "a medida do número de trabalhadores que passa pelos postos de trabalho de uma seção, empresa, setor ou ramo, num determinado período de tempo" (3). Este tipo de movimento, portanto, pode ser medido através de procedimentos operativos. Os resultados obtidos podem ser caracterizados de acordo com as repercussões ao nível da eficiência e da produtividade do setor ou da empresa. Esta avaliação determinará as intervenções a serem feitas no sentido de controlar a rotatividade.

$\mathrm{Na}$ enfermagem, o estudo pioneiro acerca da rotatividade na área hospitalar foi realizado nos Estados Unidos, pela Fundação Kellog, em 1950 (4). Os resultados obtidos nesse estudo apontaram para uma rotatividade efetiva média da força de trabalho de $63 \%$ no ano de estudo. Os custos diretos envolvidos nessa movimentação foram da ordem de US\$ 40653 para todos os departamentos estudados; o departamento de enfermagem respondeu por metade desses custos. Segundo este estudo, as taxas de rotatividade atendem ao propósito de verificar e controlar a mobilidade da força de trabalho e servem como indicativo da estabilidade desses trabalhadores no emprego, além de se constituírem num instrumento administrativo efetivo (4).

Inúmeros são os estudos norte-americanos desenvolvidos ao longo das últimas décadas sobre rotatividade no setor hospitalar (5-14). A preocupação evidenciada nestes trabalhos é com a viabilidade financeira das instituições, uma vez que, ao longo do tempo, parcelas significativas de seus orçamentos estavam sendo destinadas ao processo de reposição de enfermeiros e pessoal de enfermagem, o que caracteriza a rotatividade como uma questão econômica. Estes estudos, portanto, tinham 
como finalidade o controle, a manutenção ou a elevação da produtividade e utilizaram a mensuração da rotatividade como um dos indicadores para tais análises.

Na enfermagem brasileira, raros são os estudos acerca dessa temática. Entre eles, destaca-se como pioneiro o levantamento de recursos e necessidades de enfermagem no Brasil desenvolvido pela Associação Brasileira de Enfermagem entre 1956 e 1958 (15) e publicado em 1980. Além deste, podem ser citados outros dois estudos, ${ }^{2}$ que analisaram a rotatividade no âmbito de um hospital-escola e restringem-se à categoria dos enfermeiros. O que se observa, porém, é que, desde o final da década de 70 e no decorrer dos anos 80, vários estudos e reflexões acerca do processo de trabalho, do mercado e da força de trabalho em enfermagem (FTE) vêm emergindo (16-18). Estes buscam situar a prática da enfermagem em sua historicidade, considerando-a nas suas articulações com os demais processos de trabalho na área da saúde. Ao abordarem as condições de trabalho do pessoal de enfermagem, os estudos evidenciam uma situação de alta rotatividade. Entretanto, este movimento ainda carece de investigação mais aprofundada. Essa percepção, aliada ao contato com enfermeiros de hospitais que referem freqüentemente uma alta rotatividade no setor de enfermagem, gerou o presente estudo, cujo objetivo é medir e analisar a estrutura da rotatividade dos trabalhadores de enfermagem nos hospitais do Município de Ribeirão Preto.

\section{MATERIAIS E MÉTODOS}

O presente estudo foi realizado em 1990, na rede hospitalar geral do Município de Ribeirão Preto, localizado na região nordeste do Estado de São Paulo. Os nove hospitais gerais envolvidos na pesquisa foram classificados e codificados da seguinte forma: um hospital público (A); três filantrópicos (B-D); e cinco privados (E-I). A classificação adotada é aquela proposta pela unidade de avaliação e controle (UAC) do Escritório Regional de Saúde 50, do Município de Ribeirão Preto, segundo categoria de financiamento junto ao Sistema Único de Saúde (SUS). Cabe salientar que em 1990, momento da realização desde estudo, o sistema de saúde brasileiro passou por um processo de reorganização em decorrência da criação do

\footnotetext{
2 Anselmi ML. "Estudo da rotatividade dos enfermeiros de um hospital-escola" [dissertação de mestrado]. Ribeirão Preto: Escola de Enfermagem da Universidade de São Paulo; 1988; e Aquino KS. "Fatores geradores da rotatividade dos enfermeiros de um hospital geral governamental da cidade de São Paulo" [dissertação de mestrado]. São Paulo: Faculdade São Camilo de Administração Hospitalar; 1991.
}

SUS, que busca colocar em prática os princípios delineados pela Constituição Brasileira de 1988 e ratificados na Lei Orgânica da Saúde (No. 8080/1990). Estes princípios podem ser assim sintetizados: 1) unificação institucional dos serviços de saúde sob a administração do Ministério da Saúde; 2) descentralização dos serviços de saúde; 3) participação popular nos serviços de saúde; e 4) reorganização das estruturas que prestam serviços de saúde em uma rede integrada e hierarquizada segundo complexidade e especialização, articulada nos níveis primário, secundário e terciário. Os hospitais são a estrutura de referência para ações de alta complexidade, responsabilidade do nível terciário. A implementação do SUS teve como base a transferência para os municípios dos recursos e da responsabilidade de gestão dos sistemas de saúde.

As instituiçõoes estudadas contavam, em 1989, com um total de 1833 leitos. Em 1990, o número total de leitos era de 1761 , distribuídos da seguinte forma: 697 leitos no hospital público; 537 leitos nos hospitais filantrópicos; e 527 nos hospitais privados. Em 1989, foram efetuadas 78502 internações e, em 1990, 85 657. Do total de internações, 65,7\% foram realizadas através do SUS, o que significa que a assistência à saúde no âmbito terciário foi efetivada fundamentalmente com recursos originários do setor público. Segundo dados do centro de processamento de dados hospitalares do Departamento de Medicina Social da Faculdade de Medicina de Ribeirão Preto (Universidade de São Paulo), a faixa média de ocupação nos hospitais filantrópicos foi de 42,9\% em 1989; e de 58,5\% em 1990. Nos hospitais privados, a taxa média de ocupação foi de 39,8\% em 1989; e de 43,4\% em 1990. No hospital público este indicador apresentou valores de 78,1\% em 1989 e de 76,1\% em 1990.

A população investigada constituiu-se de todos os trabalhadores de enfermagem, em suas diferentes categorias, a saber: enfermeiro, auxiliar de enfermagem, técnico de enfermagem e atendente de enfermagem. Foram considerados os trabalhadores que em $1^{\circ}$ de janeiro de 1990 estavam vinculados através de contrato de trabalho às instituições hospitalares estudadas e aqueles que no decorrer do ano (até 31 de dezembro de 1990) ingressaram ou se desligaram formalmente das instituições.

Esse grupo de trabalhadores foi acompanhado durante o ano de 1990 para verificar saída ou permanência no emprego. A verificação foi feita através de consulta mensal ao cadastro geral de empregados e desempregados (lei $\mathrm{n}^{\circ}$ 4923/65 do Ministério do Trabalho) junto à seção de pessoal de cada instituição. O cadastro, que se constituiu no instrumento de coleta dos dados da pesquisa, registra as admissões e demissões ocorridas durante 
cada mês, bem como categoria do trabalhador, data de admissão ou demissão e outros dados.

Para mensurar a rotatividade, dois indicadores foram selecionados: a taxa líquida de substituição, preconizada por Tanabe, ${ }^{3}$ e a mediana de tempo de serviço dos demitentes, conforme Duxbury e Armstrong (19). A taxa líquida de substituição (TLS) é dada por $100\left(\mathrm{~L}_{-1}-\mathrm{Lf}\right)$, onde $\mathrm{L}_{-1}$ representa o número de empregados da categoria com menos de um ano de serviço ao final do período analisado, e Lf representa o número total de empregados da categoria em serviço no final do período analisado. Este indicador, que representa a substituição média de pessoal no período, tem a vantagem de eliminar a contagem sucessiva de trabalhadores em uma mesma vaga, ou seja: mesmo que mais de um trabalhador tenha ocupado a vaga durante o período analisado, só é computada na aplicação do indicador a admissão do trabalhador que ainda ocupar a vaga no final do período, no caso, 31 de dezembro de 1990. Portanto, para cada vaga, só uma admissão é levada em conta no período analisado.

A mediana do tempo de serviço (MTS) dos demitentes é expressa pelo período, em anos ou meses, no qual metade do grupo de trabalhadores desligados das instituições pesquisadas durante $o$ ano de 1990 deixou o trabalho, levando em conta o tempo de serviço de cada um, enquanto a outra metade continuou trabalhando. Assim, através desse indicador é possível localizar com que "tempo típico de serviço" um grupo de trabalhadores está deixando a instituição e, deste modo, se a rotatividade está centrada nos trabalhadores mais antigos ou naqueles que estão há pouco tempo no emprego. Valores baixos para a mediana indicam rotatividade elevada.

\section{RESULTADOS E DISCUSSÃO}

A freqüência absoluta de admissões e desligamentos já aponta o nível de movimentação da FTE. A tabela 1 apresenta a distribuição do quantitativo de trabalhadores de enfermagem, por categoria, alocados nos hospitais em janeiro de 1990. Pela tabela 2 verificamos 673 admissões e 565 desligamentos durante o ano de 1990, o que caracteriza, de modo geral, um incremento no número de trabalhadores na área hospitalar de Ribeirão Preto.

A maior movimentação ocorreu nos hospitais filantrópicos. O hospital D chama a atenção pelo

\footnotetext{
3 Tanabe M. "Contribuição ao estudo da rotatividade da mão-deobra" [tese de doutorado]. São Paulo: Faculdade de Economia e Administração da Universidade de São Paulo; 1985.
}

fato de que, ao final do ano, 21 postos de trabalho não haviam sido preenchidos, enquanto que no hospital B houve uma ampliação efetiva de 57 postos. Nos hospitais privados também foi observado um crescimento no número de postos de trabalho, por exemplo, no hospital E. Porém, pelos dados, estes postos parecem ter sido ocupados pela categoria "atendente", em substituição aos "auxiliares de enfermagem". No hospital H, ocorreram 28 desligamentos de auxiliares de enfermagem e apenas 14 admissões. Pela quantidade de atendentes admitidos, um total de 36 , é possível supor também que o hospital tenha optado pela substituição de categorias, de uma com melhor qualificação por aquela que não dispõe de preparo formal para o exercício da enfermagem.

No hospital público A, a diferença entre admissões e desligamentos, 17 trabalhadores, não expressa grandes alterações no quadro geral. Porém, tem seu significado no aspecto do tempo que a instituição permanece com a vaga nãopreenchida, ou seja, no tempo de ausência efetiva do trabalhador (Anselmi ML, ver nota de pé de página No. 2). Por se tratar de instituição pública, os trâmites legais e burocrático-administrativos para contratação e reposição de trabalhadores são demorados.

Os dados da tabela 2 mostram que, de modo geral para todos os hospitais, com exceção dos hospitais $\mathrm{D}, \mathrm{H}$ e I, houve um número maior de admissões e que estas ocorreram preponderantemente na categoria dos atendentes e, em menor quantidade, na categoria de enfermeiros. Os hospitais D, H e I não repuseram suas perdas e, ao final do período, apresentaram déficit no número de trabalhadores. Isto pode significar que nestes hospitais as atividades de enfermagem foram desenvolvidas, durante o período, por um número menor de pessoas, já que o número de internações aumentou de 78502 em 1989 para 85657 em 1990.

\section{Taxa líquida de substituição}

Esta taxa requer uma interpretação cuidadosa. Uma TLS elevada pode significar que as substituições se processaram de forma mais intensa (alta rotatividade); porém, a TLS elevada pode também significar que houve retenção dos trabalhadores admitidos na instituição. Se a taxa é baixa, ela pode ter decorrido de um baixo nível de movimentação da força de trabalho, ou ainda revelar que a instituição, mesmo tendo admitido muitos trabalhadores ao longo do ano, permaneceu com um quantitativo reduzido destes até o final do ano, o que evidencia, neste caso, dificuldades em reter a força de trabalho. 
TABELA 1. Distribuição dos trabalhadores de enfermagem nos hospitais do município de Ribeirão Preto, segundo categoria, $1^{\circ}$ de janeiro de 1990

\begin{tabular}{|c|c|c|c|c|c|c|}
\hline \multirow[b]{2}{*}{ Hospital } & \multirow[b]{2}{*}{ Código } & \multicolumn{5}{|c|}{ Categoria } \\
\hline & & Atendente & Auxiliar & Técnico & Enfermeiro & Total \\
\hline Público & A & 458 & 624 & 60 & 278 & 1420 \\
\hline \multirow{3}{*}{ Filantrópico } & B & 134 & 93 & 52 & 21 & 300 \\
\hline & C & 106 & 10 & 3 & 2 & 121 \\
\hline & D & 119 & 21 & 10 & 3 & 153 \\
\hline Subtotal & & 359 & 124 & 65 & 26 & 574 \\
\hline \multirow[t]{5}{*}{ Privado } & $E$ & 102 & 79 & 31 & 26 & 238 \\
\hline & $\mathrm{F}$ & 27 & 41 & 21 & 8 & 97 \\
\hline & G & 15 & 2 & - & 2 & 19 \\
\hline & $\mathrm{H}$ & 54 & 63 & - & 17 & 134 \\
\hline & 1 & 47 & 13 & 13 & 11 & 84 \\
\hline Subtotal & & 245 & 198 & 65 & 64 & 572 \\
\hline Total & & 1062 & 946 & 190 & 368 & 2566 \\
\hline
\end{tabular}

Fonte: Seção de pessoal dos hospitais e cadastro geral de empregados e desempregados (lei no 4923/65 do Ministério do Trabalho).

TABELA 2. Freqüência absoluta de admissões (A) e desligamentos (D) dos trabalhadores de enfermagem nos hospitais do Município de Ribeirão Preto, segundo categoria, 1990

\begin{tabular}{|c|c|c|c|c|c|c|c|c|c|c|c|}
\hline \multirow[b]{3}{*}{ Hospital } & \multirow[b]{3}{*}{ Código } & \multicolumn{10}{|c|}{ Categoria } \\
\hline & & \multicolumn{2}{|c|}{ Atendente } & \multicolumn{2}{|c|}{ Auxiliar } & \multicolumn{2}{|c|}{ Técnico } & \multicolumn{2}{|c|}{ Enfermeiro } & \multicolumn{2}{|c|}{ Total } \\
\hline & & A & $D$ & A & $D$ & $A$ & $\mathrm{D}$ & A & $D$ & A & $D$ \\
\hline Público & A & - & 21 & 54 & 32 & 1 & 2 & 55 & 38 & 110 & 93 \\
\hline Filantrópico & $B$ & 97 & 36 & 15 & 20 & 8 & 12 & 17 & 12 & 137 & 80 \\
\hline & C & 68 & 65 & 7 & 7 & 4 & 4 & 15 & 9 & 94 & 85 \\
\hline & D & 55 & 64 & 3 & 15 & 1 & 2 & 4 & 3 & 63 & 84 \\
\hline Subtotal & & 220 & 165 & 25 & 42 & 13 & 18 & 36 & 24 & 294 & 249 \\
\hline Privado & E & 65 & 40 & 29 & 29 & 17 & 13 & 11 & 10 & 122 & 92 \\
\hline & $E$ & 3 & 3 & 20 & 15 & 11 & 7 & 6 & 5 & 40 & 30 \\
\hline & G & 24 & 17 & 5 & 3 & - & - & 2 & 1 & 31 & 21 \\
\hline & $\mathrm{H}$ & 36 & 24 & 14 & 28 & - & - & 4 & 5 & 54 & 57 \\
\hline & 1 & 11 & 11 & 7 & 9 & 2 & - & 2 & 3 & 22 & 23 \\
\hline Subtotal & & 139 & 95 & 75 & 84 & 30 & 20 & 25 & 24 & 269 & 223 \\
\hline Total & & 359 & 281 & 154 & 158 & 44 & 40 & 116 & 86 & 673 & 565 \\
\hline
\end{tabular}

Fonte: Seção de pessoal dos hospitais e cadastro geral de empregados e desempregados (lei $n^{0} 4923 / 65$ do Ministério do Trabalho).

Assim, diante de qualquer resultado obtido na aplicação deste indicador, faz-se necessário associar outras informações, como número de admissões e desligamentos, pessoal alocado no início e final do período, afim de que as análises desenvolvidas contemplem a abrangência e possíveis contradições do indicador. Na tabela 3, dos nove hospitais estudados, a maior TLS (62\%) ocorreu no setor privado, no hospital G, indicando intensa substituição dos postos de trabalho e, conseqüentemente, uma alta rotatividade.

A escassez de estudos nacionais sobre rotatividade na área de enfermagem, principalmente no tocante à quantificação, torna difícil definir o que é nível elevado de rotatividade. Recorremos, portanto, à produção norte-americana, a qual poderia dar sustentação à análise dos resultados obtidos neste estudo. As taxas médias de rotatividade acima de $26 \%(13,14)$ implicam em altos custos, produzindo impactos financeiros adversos tanto para o departamento de enfermagem quanto para o hospital e para o próprio sistema de assistência à saúde. Outro estudo (8) considera taxas acima de $30 \%$ pouco aceitáveis. Ainda de acordo com o mesmo estudo, quando as taxas chegam a $50 \%$ provocam custos elevados no que se refere ao processo de reposição de trabalhadores e possivelmente comprometem a produtividade. Sob esta perspectiva, pelos resultados contidos na tabela 3 , evidencia-se que, de modo geral, a rotatividade foi elevada na rede hospitalar de Ribeirão Preto. 
TABELA 3. Taxa líquida de substituição (TLS) a dos trabalhadores de enfermagem nos hospitais do Município de Ribeirão Preto, segundo categoria, 1990

\begin{tabular}{|c|c|c|c|c|c|c|}
\hline \multirow[b]{2}{*}{ Hospital } & \multirow[b]{2}{*}{ Código } & \multicolumn{5}{|c|}{ Categoria } \\
\hline & & Atendente & Auxiliar & Técnico & Enfermeiro & Total \\
\hline Público & A & - & 7 & 2 & 15 & 6 \\
\hline \multirow[t]{3}{*}{ Filantrópico } & B & 43 & 14 & 12 & 46 & 41 \\
\hline & $C$ & 38 & 30 & 33 & 100 & 32 \\
\hline & D & 24 & 11 & - & 25 & 22 \\
\hline Subtotal & & 40 & 15 & 12 & 55 & 32 \\
\hline \multirow{5}{*}{ Privado } & $E$ & 39 & 29 & 40 & 33 & 36 \\
\hline & $\mathrm{E}$ & 11 & 30 & 32 & 55 & 27 \\
\hline & G & 59 & 100 & - & 33 & 62 \\
\hline & $\mathrm{H}$ & 41 & 16 & - & 19 & 29 \\
\hline & 1 & 17 & 36 & 13 & 20 & 19 \\
\hline Subtotal & & 35 & 52 & 32 & 31 & 39 \\
\hline Total & & 22 & 12 & 17 & 22 & 18 \\
\hline
\end{tabular}

${ }^{a} T L S=100\left(L_{-1}-L f\right) ; L_{-1}=$ número de empregados da categoria com menos de um ano de serviço ao final do período analisado; $L f$ = número total de empregados da categoria em serviço no final do período analisado.

Em relação ao hospital D, cabem algumas análises particularizadas. A sua TLS foi de 21,96\%, relativamente baixa quando comparada aos demais hospitais. É necessário apontar que, durante o ano, a instituição apresentou um número de desligamentos de 84 , maior do que o número de admissões, de 63 (tabela 2). Destas admissões, apenas 29 funcionários foram mantidos no emprego. Logo, não houve substituição efetiva de trabalhadores, uma vez que 21 postos permaneceram vagos e o quadro geral para todas as categorias mostrou-se defasado em relação ao quantitativo existente no início de 1990.

Nos hospitais filantrópicos, as categorias mais substituídas foram os atendentes e os enfermeiros; no setor privado é possível caracterizar que houve renovação expressiva de trabalhadores, acima de $30 \%$ em todas as categorias.

As menores taxas foram verificadas no hospital público A, sendo os enfermeiros os mais substituídos. Em estudo realizado no período de 1979 a 1985 (Anselmi ML, ver nota de pé de página ${ }^{\circ}$ 2), esta mesma instituição já mostrava uma substituição elevada para esta categoria, o que parece ter persistido no ano de 1990. Nos resultados por categoria, atendente e enfermeiro foram as categorias mais substituídas na rede hospitalar de Ribeirão Preto.

\section{Mediana de tempo de serviço dos demitentes}

Na tabela 4 observamos que o hospital público A apresenta a maior MTS. Entretanto, a mediana de 1 ano de serviço para os auxiliares de enfermagem demitentes desse hospital pode ser considerada baixa quando comparada às demais categorias, significando uma alta rotatividade para essa categoria no hospital A. Nos hospitais filantrópicos e privados, a MTS apresentou valores baixos, 8 meses e 1 ano, respectivamente.

Nos resultados por categoria, observa-se baixa MTS para atendentes, auxiliares e enfermeiros. Para todo o conjunto de trabalhadores que se desligaram em 1990 (565), a MTS foi de 11 meses, portanto inferior a um ano, caracterizando que a rotatividade foi elevada na área hospitalar. Nos hospitais filantrópicos, observou-se que para as categorias de atendente e enfermeiro a MTS variou de 2 a 8 meses. Neste caso, pode-se entender que a rotatividade esteve predominantemente centrada nos empregos novos (funcionários ingressados há pouco tempo na instituição). Considerando que a cada desligamento corresponde uma nova demissão, nos hospitais B e C, onde o número de admissões em 1990 superou o de demissões, é possível supor que uma mesma vaga tenha sido ocupada, ao longo do ano, por diferentes trabalhadores. No caso do hospital C, a taxa de MTS para enfermeiros foi de 2 meses e o número de admissões nesta categoria foi de 15. Portanto, é possível supor que a cada 2 meses um novo enfermeiro foi admitido neste hospital.

De modo geral, para o ano de 1990, em Ribeirão Preto, observou-se um incremento da FTE hospitalar na ordem de $4,2 \%$. A categoria de enfermeiro foi a que apresentou maior crescimento $(8 \%)$ seguida pelos atendentes $(7 \%)$, que preencheram postos de trabalho vacantes deixados pelos auxiliares, uma vez que esta categoria apresentou redução de $0,42 \%$. Os auxiliares demitentes dos hospitais filantrópicos e privados podem ter sido absorvidos pelo setor público, seja pelo hospital A, 
TABELA 4. Mediana de tempo de serviço, em meses, dos trabalhadores de enfermagem demitentes nos hospitais do Município de Ribeirão Preto segundo categoria, 1990

\begin{tabular}{|c|c|c|c|c|c|c|}
\hline \multirow[b]{2}{*}{ Hospital } & \multirow[b]{2}{*}{ Código } & \multicolumn{5}{|c|}{ Categoria } \\
\hline & & Atendente & Auxiliar & Técnico & Enfermeiro & Total \\
\hline Público & A & 90 & 12 & 180 & 38 & 42 \\
\hline \multirow[t]{3}{*}{ Filantrópico } & B & 6 & 54 & 52 & 6 & 10 \\
\hline & $C$ & 7 & 11 & 2 & 2 & 6 \\
\hline & $D$ & 8 & 19 & 24 & 7 & 9 \\
\hline Subtotal & & 7 & 24 & 36 & 3 & 8 \\
\hline \multirow[t]{5}{*}{ Privado } & $E$ & 9 & 18 & 20 & 23 & 15 \\
\hline & E & 156 & 7 & 18 & 7 & 15 \\
\hline & G & 7 & 15 & - & 5 & 7 \\
\hline & $\mathrm{H}$ & 12 & 24 & - & 27 & 17 \\
\hline & 1 & 11 & 7 & - & 21 & 11 \\
\hline Subtotal & & 10 & 15 & 21 & 20 & 12 \\
\hline Total & & 9 & 17 & 26 & 16 & 11 \\
\hline
\end{tabular}

que não contrata mais atendentes (conforme a Resolução COFEN n ${ }^{\circ} 96$ de $1987^{4}$ ), ou, ainda, pela rede básica de serviços de saúde do município. Nestes últimos anos, com o processo de municipalização, o serviço municipal de saúde vem se reorganizando, ampliando sua capacidade de atendimento e com isto gerando demanda por pessoal qualificado, como auxiliares e enfermeiros. Entre 1988 e 1990, a Secretaria Municipal de Saúde ampliou o quantitativo de auxiliares em 101\% e o de enfermeiros, em $204 \% .^{5}$ Portanto, a rotatividade dessas categorias na rede hospitalar pode ser explicada, em parte, por todo o processo de reorganização do setor público.

$O$ crescimento da FTE no setor hospitalar articula-se ao movimento contínuo e acelerado de expansão que o mercado de trabalho em saúde e sua força de trabalho vêm apresentando nesta última década. ${ }^{6}$ Este movimento é determinado pela crescente demanda por serviços de saúde, onde o Estado cria demanda efetiva para o setor privado, através de subsídios médicos, incentivos tributários, contratos e convênios com empresas e grupos médicos, indústrias farmacêuticas e de equipamentos, e estimula o crescimento de emprego aos trabalhadores de saúde fora do âmbito estatal (20).

\footnotetext{
4 De acordo com esta resolução, a partir de janeiro de 1989 os atendentes que não possuem autorização do COFEN para o exercício da profissão estão sujeitos à interrupção do contrato de trabalho ou à transferência de área de atividade nas instituições de saúde. A resolução aplica-se a todas as instituições de saúde, mas tem sido respeitada apenas nas instituições públicas. Nestas instituições, os atendentes demitidos são substituídos por auxiliares de enfermagem.

5 Almeida MCP. “O trabalho da enfermagem e sua articulação com o processo de trabalho em saúde coletiva na rede básica de saúde de Ribeirão Preto" [tese de livre-docência]. Ribeirão Preto: Escola de Enfermagem de Ribeirão Preto, Universidade de São Paulo; 1991.

6 Girardi SN. "Elementos da dinâmica e estrutura do emprego em saúde no Brasil 1974-1984" [mimeografado]. Belo Horizonte, 1989.
}

Os resultados obtidos na quantificação da rotatividade pelo presente estudo permitem concluir que este movimento foi concreto na FTE hospitalar de Ribeirão Preto. Os valores mostram ainda uma acentuada e intensa substituição de trabalhadores nas instituições filantrópicas. Estas, pela articulação e dependência com o setor público, acabam por colocá-lo como um parceiro nos custos e riscos à produtividade, elementos estes provavelmente determinados, em parte, pela elevada rotatividade da FTE.

Além disso, de acordo com os níveis registrados em 1990, a rotatividade poderia estar dificultando o processo de concretização do SUS, uma vez que apenas o crescimento da força de trabalho, embora importante, por si só não é capaz de assegurar a implantação desse sistema. Juntamente com outros elementos políticos, econômicos e organizacionais, é a permanência no trabalho de sujeitos que são atores sociais dotados de ideologias e interesses próprios - capazes, portanto, de defender determinadas políticas - que estabelece possibilidades de viabilizar o SUS.

No tocante aos hospitais privados onde a lógica predominante é a de mercado, em que a produtividade deve traduzir matematicamente o uso mais eficiente entre capital e trabalho na busca de maximização dos lucros e minimização dos custos, o trabalhador, assim como os demais recursos produtivos, pode ser manipulado no sentido de conter ou estimular os desligamentos, conforme estes afetem a produtividade organizacional. A rotatividade da FTE encontrada nestas instituições, para algumas em níveis comprometedores, leva-nos a refletir acerca das bases ou critérios que alicerçam a produtividade. Em algumas instituições filantrópicas e privadas, o mecanismo de redução e controle 
de custos foi supostamente a própria rotatividade, com a substituição de categorias mais qualificadas, com salários maiores, por categorias sem preparo formal e de menor custo, como os atendentes.

A intervenção e modificação dos níveis de rotatividade dependerá, essencialmente, de ações políticas que busquem compreender, no processo de construção e reorganização do sistema de saúde, qual o papel a ser ocupado pelos recursos humanos, em qual modelo assistencial e sob que prática de gerência. Através dessa reflexão, os recursos humanos poderão vir a ser não apenas instrumentos de produção de serviços, mas agentes capazes de promover autonomia, ainda que relativa, no encaminhamento de políticas que venham a produzir mudanças positivas na prestação de serviços de saúde.

\section{REFERÊNCIAS}

1. Nogueira RP. A força de trabalho em saúde. Rev Administraçao Publica 1983; 17(3):61-70.

2. Nogueira RP. El proceso de producción de servicios de salud. Educ Med Salud 1991;25(1):15-27.

3. Departamento Intersindical de Estatística e Estudos Sócio-Econômicos (DIEESE). Rotatividade: conceito e determinantes (primeira parte). Bol DIEESE 1988;7:7-19. (Estudos e pesquisas).

4. Sturdavant M, Hitt D, Jydstrup RA. A study of turnover and its costs. Hospitals 1955;29(5):59-62.

5. Melbin M, Taub DL. The high cost of replacing a nurse. Hospitals 1966;40 (16):112-122.

6. Tsui A. Diagnosis of turnover can convert causes to assets. Hospitals 1977;51 (4):157-162.

7. Wolf GA. Nursing turnover: some causes and solutions. Nurs Outlook 1981; 24(4):233-236.

8. Price JL, Mueller CW. Professional turnover: the case of nurses. New York: SP Medical \& Scientific Books; 1981.
9. Prescott PA, Bowen SA. Controlling nursing turnover. Nurs Management 1987;18(6):60-66.

10. Parasuraman $S$. Nursing turnover: an integrated model. Res Nurs Health 1989; 18(4):267-277.

11. Cavanagh SJ. Nursing turnover: literature review and methodological critique. J Adv Nurs 1989;14(7):587-596.

12. Cavanagh SJ. Predictors of nursing staff turnover. J Adv Nurs 1990;15(3):373-380.

13. Jones CB. Staff nurse turnover costs: part I, conceptual model. J Nurs Adm 1990;20:18-22.

14. Jones CB. Staff nurse turnover costs: part II, measurements and results. J Nurs Adm 1990;20(5):27-32.

15. Associação Brasileira de Enfermagem. Relatório final do levantamento de recursos $e$ necessidade de enfermagem no Brasil 1956-1958. Brasília: ABEn; 1980.

16. Almeida MCP, Rodrigues AM, Castellanos BEP, Leopardi da Rosa MT, Mendes SAS. A situação da enfermagem nos anos 80. Anais do Congresso Brasileiro de Enfermagem 41, Florianópolis. 1989: 43-75.
17. Leopardi da Rosa MT, Rodrigues AM, Castellanos BEP, Almeida MCP, Mendes SAS. O desenvolvimento técnicocientífico da enfermagem - uma aproximação com instrumentos de trabalho. Anais do Congresso Brasileiro de Enfermagem 41, Florianópolis. 1989:97-126.

18. Castellanos BEP, Rodrigues AM, Almeida MCP, Leopardi da Rosa MT, Mendes SAS. Os desafios da enfermagem para os anos 90. Anais do Congresso Brasileiro de Enfermagem 41, Florianópolis. 1989:147-169.

19. Duxbury ML, Armstrong GD. Calculating nurse turnover indices. J Nurs Adm 1982;12(3):18-24.

20. Medici AC, Machado MH, Nogueira RP, Girardi SN. El mercado de trabajo en salud: aspectos teóricos, conceptuales y metodologicos. Educ Med Salud 1991; 25(1):1-14.

Manuscrito aceito para publicação em 16 de agosto de 1996.

ABSTRACT The present study was carried out in 1990 with the objective of measuring and analyzing the turnover of nursing personnel in nine general hospitals located in Ribeirão Preto, São Paulo State, Brazil. The population under

Turnover of nursing personnel in hospitals in Ribeirão Preto ing attendants who started work or left their jobs between 1 January and 31 December 1990. Employment status for these workers was checked monthly based on information from the human resources department of each hospital. Two types of turnover indices were calculated: the liquid rate of substitution and the median length of service of the departing workers. Results showed that the number of nursing personnel employed in Ribeirão Preto increased in 1990. However, results also revealed a high turnover rate, especially for philanthropic and private institutions, with liquid substitution rates of $32 \%$ and $39 \%$ per year and median lengths of service of 8 months and 12 months, respectively. These circumstances could lead to higher costs and reduced productivity. The only public hospital in the study presented the lowest level of turnover (liquid substitution rate of $6 \%$ and median length of service of 42 months). Nurses and attendants had the highest substitution rates and thus were the least stable categories. 